Research Article

\title{
Application of Control Rods for Passively Suppressing Cross-Flow VIV of an Inclined Flexible Cylinder
}

\author{
Wanhai Xu (iD, ${ }^{1}$ Wenqi Qin, ${ }^{1}$ and Yan $L u \mathbb{D}^{2}$ \\ ${ }^{1}$ State Key Laboratory of Hydraulic Engineering Simulation and Safety, Tianjin University, Tianjin 300072, China \\ ${ }^{2}$ School of Civil Engineering, Tianjin University, Tianjin 300072, China \\ Correspondence should be addressed to Yan Lu; yanlu86@tju.edu.cn
}

Received 28 July 2018; Accepted 10 October 2018; Published 5 November 2018

Academic Editor: Mohammad Rafiee

Copyright (c) 2018 Wanhai Xu et al. This is an open access article distributed under the Creative Commons Attribution License, which permits unrestricted use, distribution, and reproduction in any medium, provided the original work is properly cited.

\begin{abstract}
Laboratory experiments were conducted to investigate the cross-flow (CF) vortex-induced vibration (VIV) suppression effectiveness of an inclined flexible cylinder by using control rods. 4 control rods with a diameter of $0.25 D$ (where $D$ is the diameter of the main cylinder) and outer wall distance between the control rod and the main cylinder of $0.50 D$ were attached to the main cylinder. The uniform flow was simulated by towing the cylinder models along a water tank. The corresponding flow velocity ranged from 0.05 to $1.00 \mathrm{~m} / \mathrm{s}$ with an interval of $0.05 \mathrm{~m} / \mathrm{s}$. Two inclination angle cases $\left(a=0^{\circ}\right.$ and $\left.45^{\circ}\right)$ were selected in the experiment. The inclination angle $a$ referred to the angle between the main cylinder axis and the plane orthogonal to the oncoming flow. The experimental results indicate that the control rods can effectively mitigate the CF VIV responses, such as the bending strain, displacement amplitude, dominant frequency, and dominant mode, of both the vertical $\left(a=0^{\circ}\right)$ and inclined $\left(a=45^{\circ}\right)$ flexible cylinders. The suppression effectiveness of the control rods on the $45^{\circ}$ inclined cylinder is as good as that on the vertical cylinder, and the average suppressing efficiency of the control rods is even better on the $45^{\circ}$ inclined cylinder.
\end{abstract}

\section{Introduction}

Vortex-induced vibration (VIV) of long flexible cylinder structures, such as marine risers, free spanning pipelines, and cables, is a complicated fluid-structure interaction (FSI) phenomenon in offshore engineering. The periodic forces caused by vortex shedding behind a bluff body may lead to serious structural damage and fatigue. A considerable number of researchers have investigated the mechanism and characteristics of cylinders subjected to VIV, which can be found in several comprehensive reviews [1-4]. Moreover, how to alleviate or control this kind of oscillation is one of the most challenging topics in industry and academia. Several representative devices and methods for VIV suppression have been proposed during the past few decades [5-23].

In general, to suppress the VIV is to prevent the occurrence of "lock-in" when the vortex shedding frequency is close to the dominant response frequency of the structure.
Popular methods for reducing VIV can be divided into two major types: positive control and passive control. The former one needs to rely on external energy to interfere with the flow field around the structure and disrupt the vortex shedding, e.g., a sound excitation or suction, while the passive control method is to deform the configuration of the bluff-body altering the roughness or implementing extra devices without the input of external energy. Several typical passive control devices, such as helical strakes [10-16] and control rods [17-23], have been widely recognized and applied in practical engineering situations. They show superiorities in both economic cost and VIV suppression effectiveness.

Among these devices, the control rods, which refer to smaller pipes or tubes attached around the main structure, can mitigate the lift force and vibration amplitude to a great extent by disturbing the flow field around the main cylinder. Meanwhile, the control rods will not increase the drag force and are easy to implement. Several geometrical parameters 
are vital to the suppression efficiency of the control rods, e.g., the diameter ratio of the control rod and main cylinder $(d / D$, where $d$ is the diameter of the control rod and $D$ is the diameter of the main cylinder), the number of control rods, and the spacing distance between the control rods and the main cylinder. Lee et al. [17] experimentally studied the suppression effectiveness of drag force by an upstream small control rod through varying the diameter ratio $(d / D)$ and centre-to-centre distance of the control and main cylinder $S$, and when $d / D$ was 0.233 and $S / D$ was 1.833 , the drag diminished by approximately $25 \%$ at Reynolds number of 20000. Wu et al. [19] carried out a series of experimental tests of VIV reduction of a flexible cylinder mounted with 4 control rods, and they found that the control rods could work well in mitigating the VIV response of the flexible cylinder. Silva-Ortega et al. [20] used arrangements of a circular array of 2,4 , and 8 control rods, respectively, to alleviate the VIV of the main cylinder and proved that the composition of 8 control rods mitigated the maximum oscillation amplitude by approximately $99 \%$ at $\mathrm{Re}=$ 5000-50000. Numerous numerical simulations were conducted to further investigate the suppression effectiveness of control rods. Zhang et al. [21] performed two-dimensional simulations of flow around two tandem cylinders of unequal diameter and found that the mean drag and lift on the downstream cylinder could be reduced with the diameter ratio $d / D=0.30$ and 0.50. Zhu and Yao [22] established CFD models of a cylinder with multiple control rods around and found that the arrangement of the circular array of 9 control rods could reach remarkable suppression effectiveness when $d / D=0.15$ and $S / D=0.60$. Zhu et al. [23] also numerically investigated the suppression effectiveness of 2 control rods by arranging them behind the main cylinder and found that the VIV of the main cylinder can be reduced by approximately $89 \%$ at a certain range of Reynolds numbers.

As is well known, the control rods can effectively alleviate the VIV response of the main cylinder. However, most previous research works were carried out under situations that the axis of the main cylinder is perpendicular to the direction of oncoming flow. In real engineering applications, the existence of inclination angle, $a$ (where $a$ is the angle between the main cylinder axis and the plane orthogonal to the oncoming flow), can cause secondary flow, leading to an even more comprehensive flow field interference phenomenon. Xu et al. [16] pointed out that the inclination angle has a significant influence on the suppression efficiency of another common and successful VIV suppression device, helical strakes. The VIV suppression effectiveness of helical strakes deteriorated with the increase of inclination angle. The strakes could even show negative suppression efficiency and enhanced the vibration of the inclined flexible cylinder with $a=45^{\circ}$. Based on the fact mentioned above, a series of experimental tests were designed and carried out in a towing tank to investigate the suppression effectiveness of control rods on the inclined flexible cylinder. The purpose of this paper is to experimentally check whether the control rods could achieve equivalent or even better suppression effectiveness with the vertical case when a large inclination angle exists.

\section{Experimental Setup}

The main cylinder model, which was made of a long copper pipe, covered with a smooth silicone tube, was $16.0 \mathrm{~mm}$ in diameter and $5.60 \mathrm{~m}$ in length. The corresponding aspect ratio, $L / D$ (where $L$ is the cylinder length), was 350.0. The mass per unit length of the main cylinder model was $0.3821 \mathrm{~kg} / \mathrm{m}$. The mass ratio $\left(m^{*}\right)$ of the main cylinder, which is defined as the structural mass upon the mass of displaced fluid, was 1.90. Major physical parameters of the main cylinder are listed in Table 1.

The aim of current experimental tests is to investigate the influence of inclination angle on the suppression effectiveness of the control rods rather than the optimized configuration of the control rods. Hence, a spatial arrangement of 4 control rods, as shown in Figure 1, was selected in the experiment, which has been demonstrated to be feasible in a flexible riser's VIV reduction $[18,19]$. The control rods were distributed as a circular array around the main cylinder, with a diameter ratio of $d / D=0.25$ and outer wall distance of $S=0.50 D$. Our previous research pointed out that the VIV on a $45^{\circ}$ inclined cylinder is not totally equivalent to the VIV of the vertical cylinder induced by the projection component of oncoming flow velocity in the direction perpendicular to the cylinder's axis [16]. Hence, $45^{\circ}$ is an important angle to investigate the characteristics of a VIV suppression device on the inclined cylinder.

Annular supporting devices were distributed along the main cylinder to fixate the control rods (see Figure 2(a)) and avoid movement and deformation caused by the VIV of the control rod itself. Figure 2(b) and 2(c) gives an overview of the layout of measurement points on the main cylinder. From the cross section view of the main cylinder, two sets of strain gauges were attached to the inner copper pipe to measure the bending strain responses in the cross-flow $(\mathrm{CF})$ and in-line (IL) directions, respectively. Along the main cylinder, there were seven measurement points corresponding to fourteen sets of strain gauges. During the tests, the strains were collected at a frequency of $100 \mathrm{~Hz}$. Figure 3 presents the sketch of the experimental setup. The experimental device was mounted on the towing carriage with given inclination angles $\left(a=0^{\circ}\right.$ and $\left.45^{\circ}\right)$. The cylinder model was connected to this test device by universal joints, which can provide a pinned-pinned boundary condition. The cylinder was allowed to oscillate in the CF and IL directions but was restrained from rotating. In addition, the axial tension of $450 \mathrm{~N}$ was adjusted on the cylinder model by the pulley, spring, and tensioner positioned on the supporting frame. The load cell was to monitor the axial tension on the cylinder model. Two guide planes were set at both ends of the cylinder to eliminate the boundary effect. The supporting rig was towed by a carriage at a uniform velocity, which ranged from $0.05 \mathrm{~m} / \mathrm{s}$ to $1.0 \mathrm{~m} / \mathrm{s}$ with an interval of $0.05 \mathrm{~m} / \mathrm{s}$. More details about the supporting system can be found in $[16,24]$.

After gaining the strain signals at seven measurement points, a modal analysis method, which has been successfully used in the experiments of flexible cylinders undergoing vortex shedding [25-28], was applied to reconstruct the displacement responses in our tests. The displacement of the main cylinder 
TABLE 1: Physical parameters of the main cylinder with control rods.

Outer diameter, $D$

$0.016 \mathrm{~m}$

$5.60 \mathrm{~m}$

Total length, $L$

$17.45 \mathrm{Nm}^{2}$

Bending stiffness, EI

$450 \mathrm{~N}$

Mass of the main cylinder per unit length, $m_{\mathrm{s}}$

$0.3821 \mathrm{~kg} / \mathrm{m}$

Mass ratio, $m^{*}$

1.90

Aspect ratio, $L / D$

350

Number of control rods

Number of supporting device

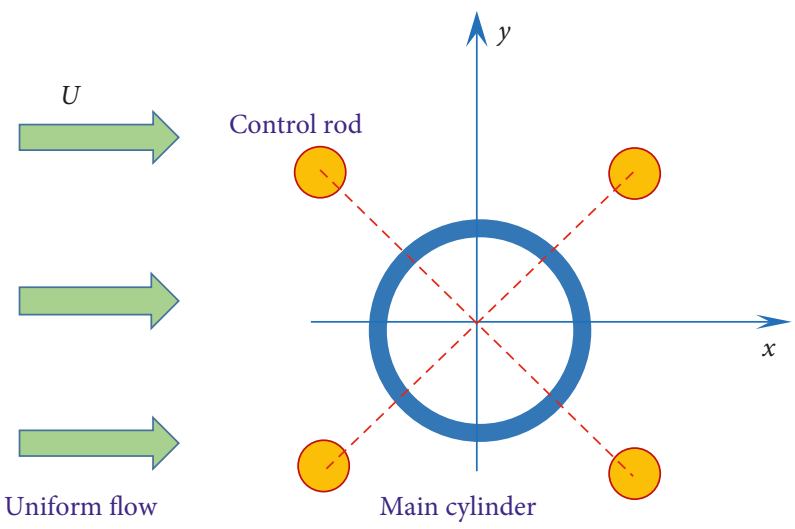

Figure 1: Schematic diagram of the main cylinder with 4 control rods.
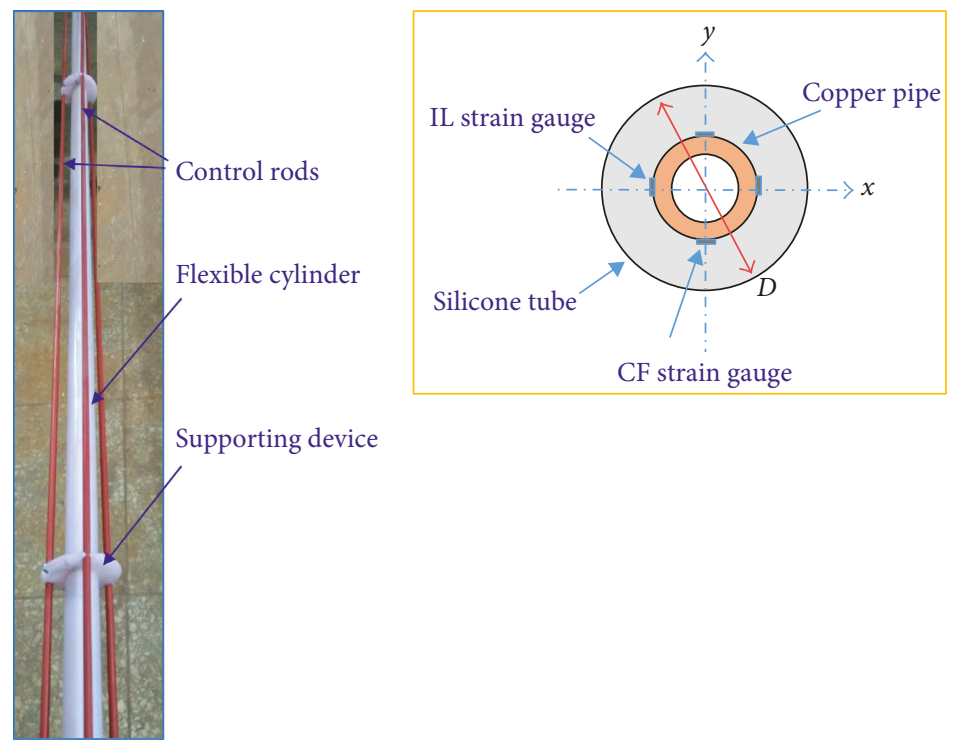

Supporting device

(a)

(b)

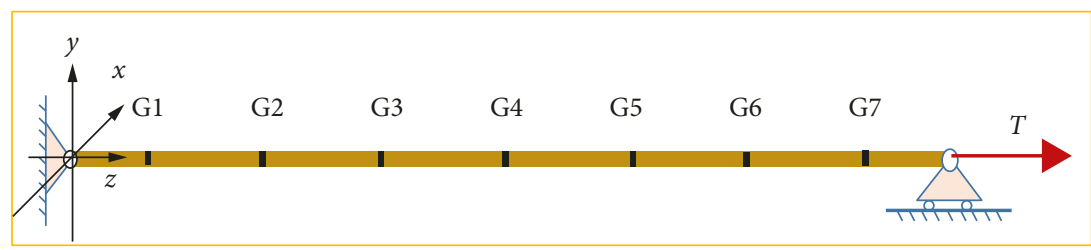

(c)

FIGURE 2: Flexible cylinder model used in the experiment. (a) Main cylinder with control rods, (b) cross section view of the main cylinder, and (c) strain gauges' positions along the main cylinder. 


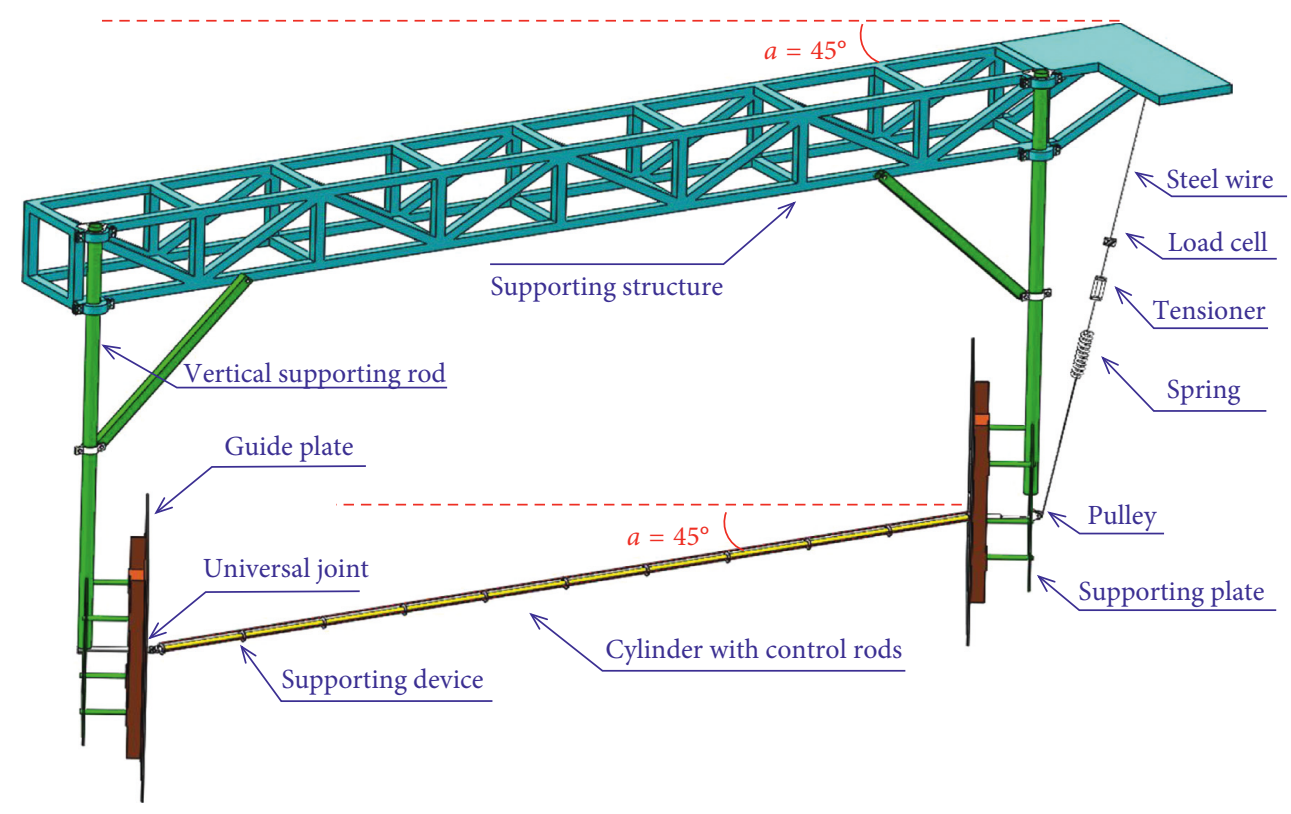

FIGURE 3: Sketch of the experimental setup.

can be assumed as the result of multimode superposition, and the CF displacement can be noted as

$$
y(z, t)=\sum_{n=1}^{\infty} w_{n}(t) \varphi_{n}(z), \quad z \in[0, L],
$$

where $y(z, t)$ is the time-varying displacement in the CF direction, $z$ represents the coordinate along the axis of the main cylinder, while $n$ is the order of vibration modes, and $w_{n}(t)$ and $\varphi_{n}(z)$ denote the modal weight and modal shape, respectively. The pinned-pinned boundary condition was applied in the experiment, and the modal shape function can be written as

$$
\varphi_{n}(z)=\sin \frac{n \pi z}{L}, \quad z \in[0, L] .
$$

Combining the relation between curvature and strain, we can finally put the formula as

$$
A W=B,
$$

herein,

$$
A=-\left(\frac{\pi}{L}\right)^{2}\left(\begin{array}{ccccc}
1^{2} \sin \frac{1 \pi z_{1}}{L} & 2^{2} \sin \frac{2 \pi z_{1}}{L} & \cdots & N^{2} \sin \frac{N \pi z_{1}}{L} \\
1^{2} \sin \frac{1 \pi z_{2}}{L} & 2^{2} \sin \frac{2 \pi z_{2}}{L} & \cdots & N^{2} \sin \frac{N \pi z_{2}}{L} \\
\vdots & \vdots & \vdots & \vdots \\
1^{2} \sin \frac{1 \pi z_{M}}{L} & 2^{2} \sin \frac{2 \pi z_{M}}{L} & \cdots & N^{2} \sin \frac{N \pi z_{M}}{L}
\end{array}\right),
$$

$W=\left[\begin{array}{llll}\omega_{1}(t) & \omega_{2}(t) & \cdots & \omega_{N}(t)\end{array}\right]^{T}$,

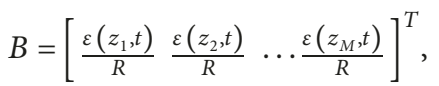

where $R$ is the outer radius of the copper pipe in the main cylinder model. Adopting a least square method to solve the formula in Equation (3), we can further obtain the value of the modal weight function, and then the spatial displacement information. The modal analysis method has been proven to be a practical way for attaining the displacement on flexible risers subjected to VIV [25-28].

\section{Results and Discussion}

3.1. VIV Suppression of the Vertical Flexible Cylinder $\left(a=0^{\circ}\right)$. The strain responses of the smooth cylinder without and with control rods under a perpendicular condition $\left(\alpha=0^{\circ}\right)$ are investigated. Figure 4 gives an example of the time history of measured strain signals at seven measurement points during 20-50 s and the corresponding spectra which were obtained by the fast Fourier transform (FFT). The flow velocity is $0.60 \mathrm{~m} / \mathrm{s}$ for this case. The top and bottom graphs show the experimental results of the smooth cylinder and the main cylinder attached with 4 control rods, respectively. It can be observed that the maximum CF strain of the vertical smooth cylinder is up to approximately $220 \mu \varepsilon$. The strain signals at the middle part of the smooth cylinder model (G4 and G5) are weaker than those on other measurement points. In the spectral plot, the CF response frequency of the smooth cylinder mainly includes two peaks. The lower component is approximately $6.2 \mathrm{~Hz}$, while the higher component is nearly $18.8 \mathrm{~Hz}$. The latter is three times the former. A similar trend was also found in the experimental study of a vertical flexible cylinder undergoing vortex shedding by Song et al. [28]. For the main cylinder attached with control rods, the strain responses present distinct mitigation. The maximum strain amplitude at different measurement points is restrained to approximately $69 \mu \varepsilon$. Also, the strain response at the middle location (G4) is considerably lower than that at other 


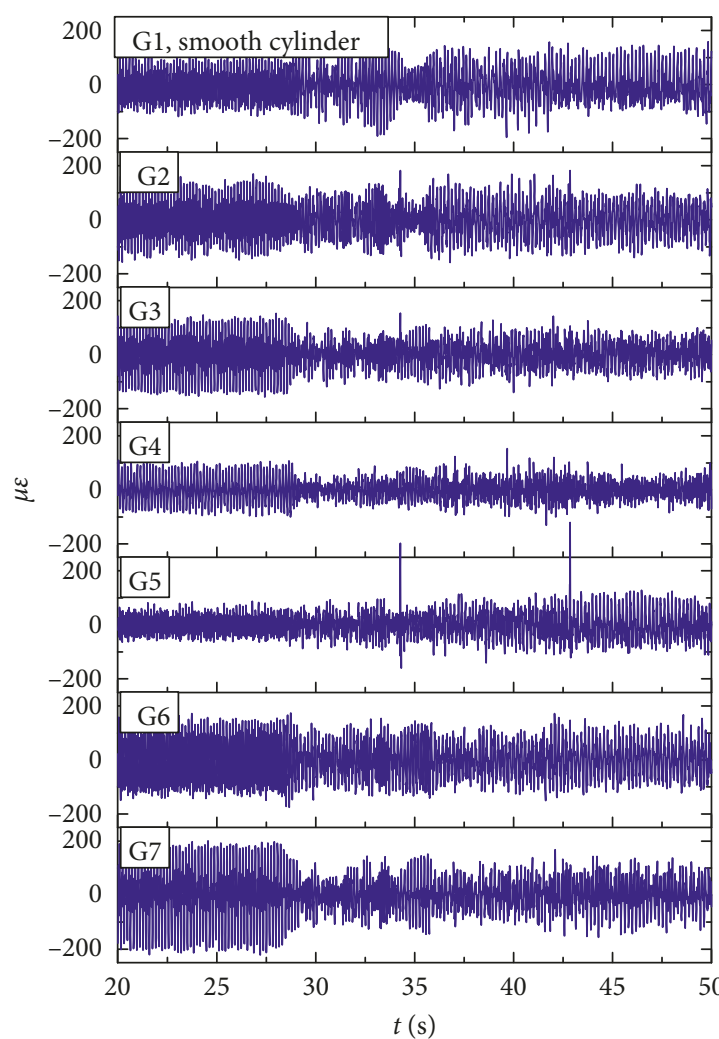

(a)

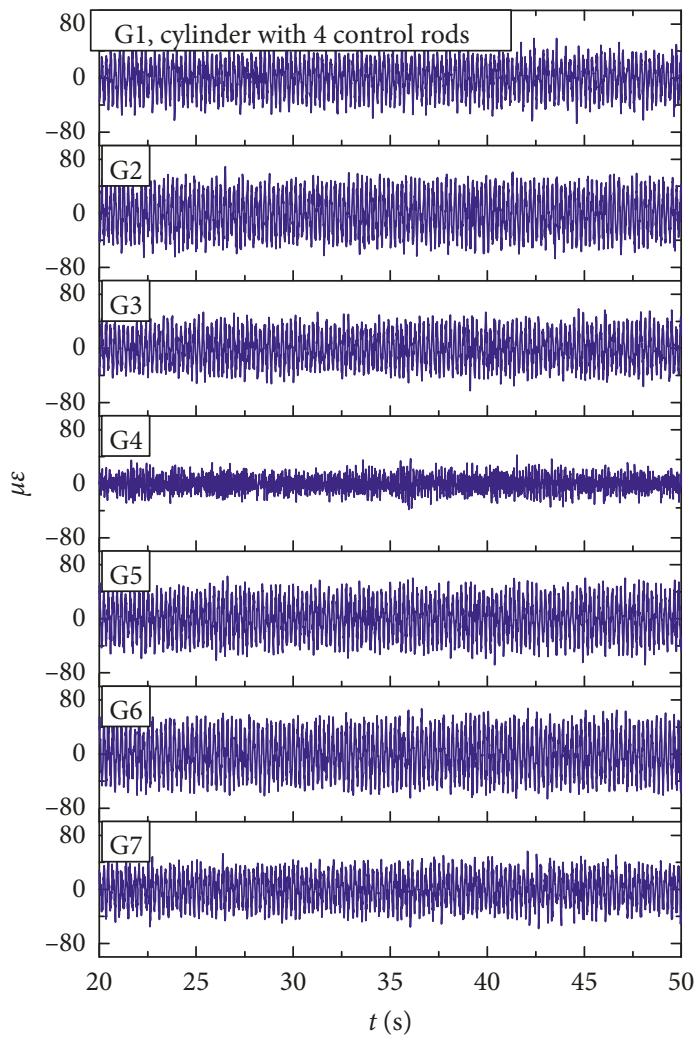

(c)

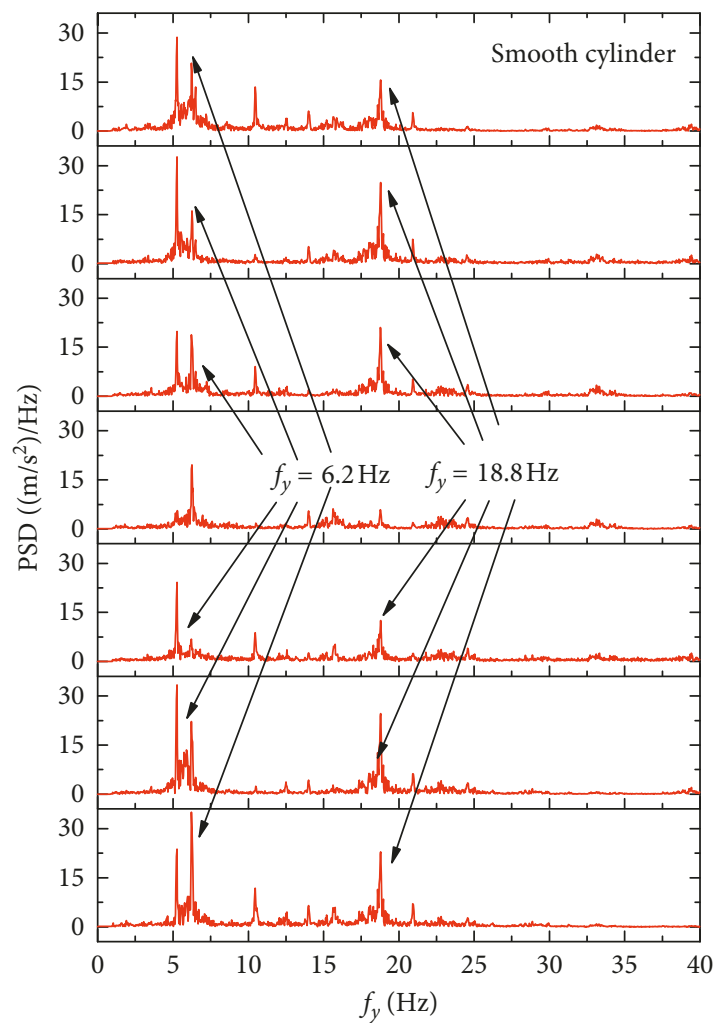

(b)

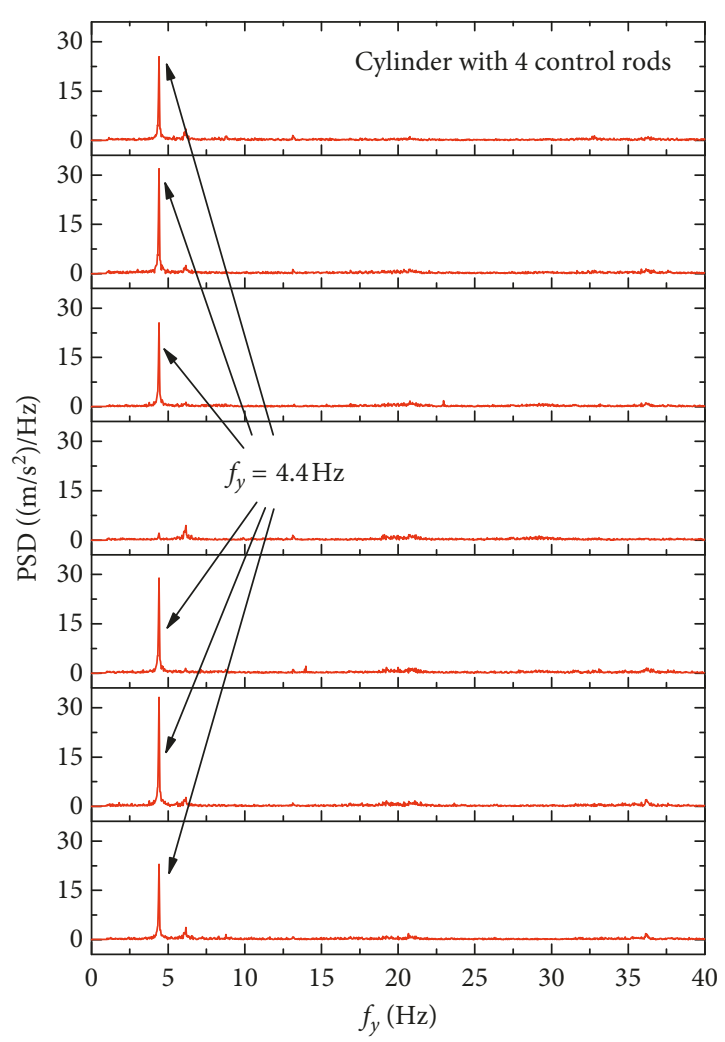

(d)

FIGURE 4: Strain response characteristic of the vertical flexible cylinder $\left(\alpha=0^{\circ}\right)$ without and with control rods undergoing vortex shedding at $U=0.60 \mathrm{~m} / \mathrm{s}$ (the first column presents time history of strain signals ((a), (c)) and the second column presents response spectra ((b), (d))). 


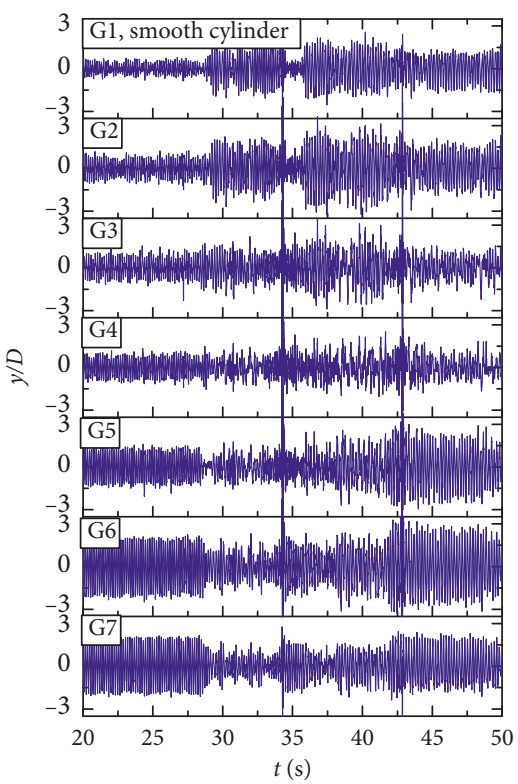

(a)

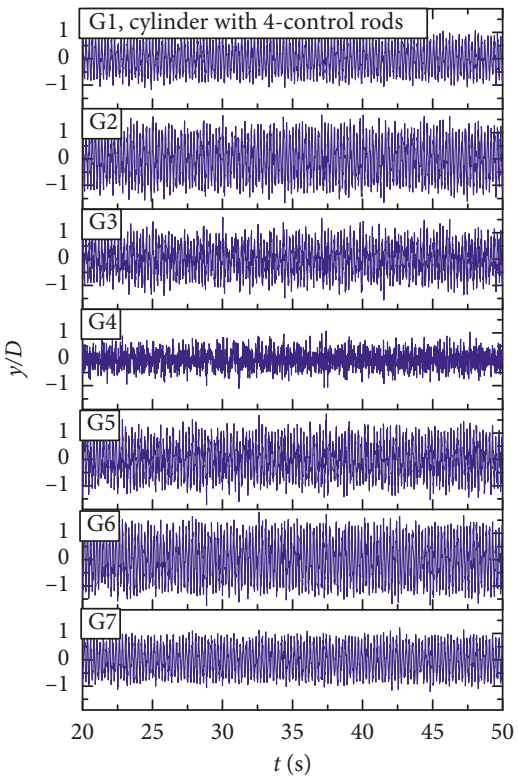

(c)

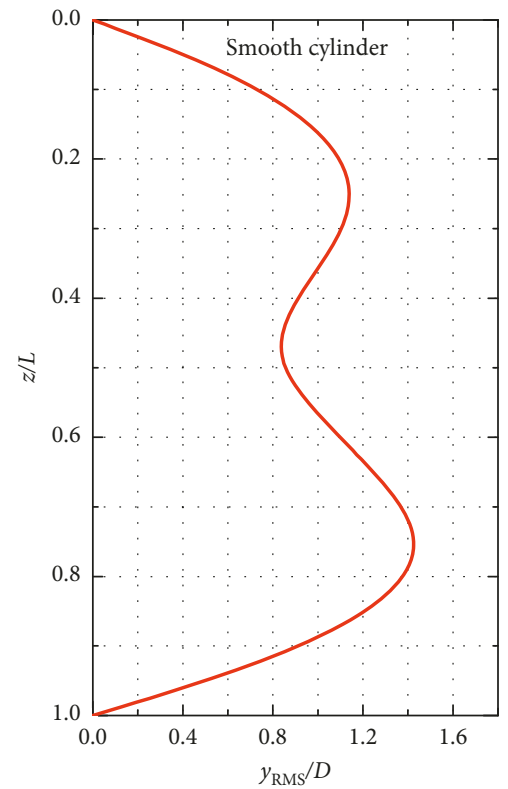

(b)

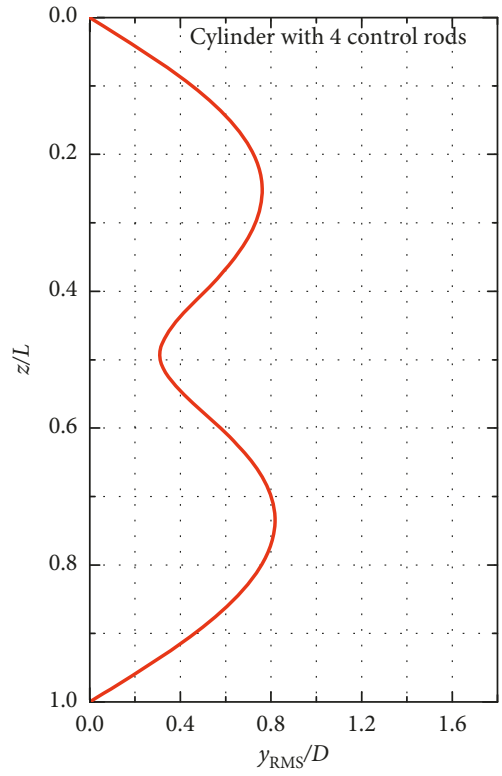

(d)

Figure 5: Displacement response characteristic of the vertical flexible cylinder $\left(\alpha=0^{\circ}\right)$ without and with control rods undergoing vortex shedding at $U=0.60 \mathrm{~m} / \mathrm{s}$ (the first column presents the time history of the nondimensional displacement ((a), (c)), the second column presents the spanwise evolutions of the RMS displacements ((b), (d))).

measurement points. Moreover, the frequency spectra only exist one peak, and the higher harmonic component disappears. The dominant frequency slightly decreases to approximately $4.4 \mathrm{~Hz}$. These experimental results indicate that the control rods can work effectively in alleviating the $\mathrm{CF}$ strain response and reducing dominant frequency of the vertical flexible cylinder. Similar findings were observed in the experimental tests of $\mathrm{Wu}$ et al. $[18,19]$.

Figure 5 demonstrates the time history and spatial distribution of the vertical flexible cylinders at $U=0.60 \mathrm{~m} / \mathrm{s}$.
The first column graph presents the time-varying displacement at the measurement points during $20-50 \mathrm{~s}$, the second column graph shows the spatial distribution of displacement along the cylinder. It is clear that the peak value in the time-varying displacement curves of the smooth cylinder can be as high as approximately $3.0 D$. It is known that standing wave and traveling wave behaviors are two important differences between the VIV responses of elastically mounted rigid cylinders and flexible ones and show that the VIV response of flexible cylinders is more 
complicated due to the response varying along the span. The response of the smooth cylinder in Figure 5 appears as a combination of traveling wave and standing wave, and the asymmetry of the distribution is observed, which can be attributed to the composition of adjacent vibration modes. The maximum root-mean-square (RMS) amplitude can reach a value of approximately $1.40 \mathrm{D}$, and the vibration is dominated by the 2 nd mode. For the main cylinder equipped with 4 control rods, the maximum displacement amplitude at seven measurement points declines to a lower level of approximately $1.70 \mathrm{D}$, occurring at locations G2 and G6 while the valley is at the middle point G4. Meanwhile, the curve in the spatial distribution plot is obviously not close to zero which indicates that the overall response is a combination of standing wave and traveling wave behaviors, and the peak value of the RMS dimensionless displacement declines to $0.80 \mathrm{D}$ as well. In addition, the CF vibration is dominated by the 2 nd mode. The experiment results above give convincing proof that the control rods can effectively mitigate the displacement responses of the vertical flexible cylinder in the CF direction, which are consistent with previous experimental and numerical studies [19, 20,23].

Figure 6 shows the maximum RMS dimensionless CF displacement of the vertical cylinder without and with control rods versus the reduced velocity. The reduced velocity herein is calculated by $V_{\mathrm{r}}=U / f_{1} D$, where $f_{1}$ is the fundamental frequency of the main cylinder in still water. For the smooth cylinder, the peak value can reach approximately $1.60 D$ when $V_{\mathrm{r}}=12.5$. The $\mathrm{CF}$ vibration amplitude valleys appear in the mode transition region. In comparison, the corresponding data on the cylinder with control rods fall to a lower level overall. When $V_{\mathrm{r}} \leq 6.26$, the displacement of the suppressed cylinder is close to that of the smooth cylinder, meaning that the VIV reduction by using control rods for the main cylinder oscillation displacement is extremely weak. With the increasing of reduced velocity, the suppression effectiveness of control rods becomes more and more notable, and the peak value of vibration amplitude for the main cylinder attached with control rods is beneath $1.0 \mathrm{D}$. Moreover, the max RMS amplitude of the suppressed cylinder is restrained to nearly zero when $V_{\mathrm{r}} \geq 20$. A similar trend was also found in suppressing VIV experiments of a flexible cylinder with 4 control rods by $\mathrm{Wu}$ et al. $[18,19]$.

Figure 7 shows the suppression efficiency of the control rods for the vertical cylinder subjected to VIV. Herein, the suppression efficiency is calculated as follows:

$$
\eta=\frac{y-y_{\mathrm{cr}}}{y} \times 100 \%
$$

where $y$ and $y_{\text {cr }}$ denote the CF displacement amplitude on the cylinder without and with control rods, respectively. It can be observed that the suppression efficiency of control rods fluctuates approximately $40 \%$ when $V_{\mathrm{r}} \leq 15$ and keeps going up as the reduced velocity increases. The suppression efficiency maintains approximately $90 \%$ when the reduced velocity is over 20 . In addition, the average efficiency is approximately $60.2 \%$, which indicates that the control rods

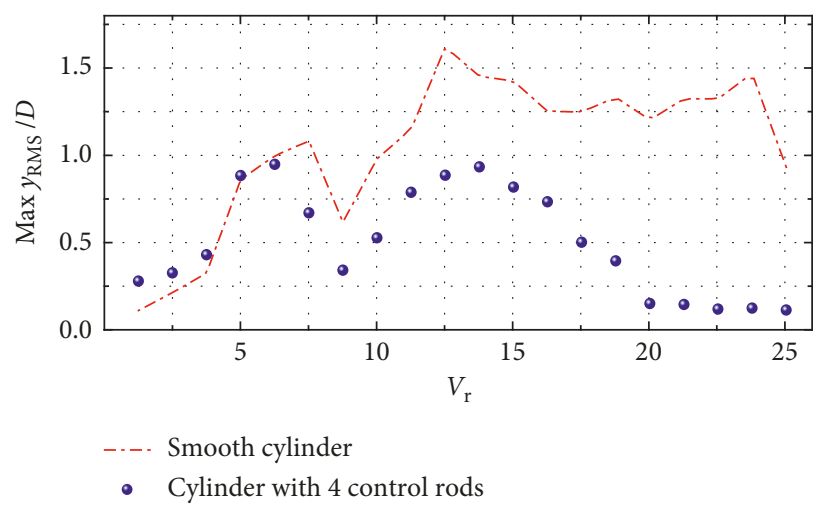

FIGURE 6: Maximum RMS dimensionless displacement of the main vertical flexible cylinder with control rods versus the reduced velocity.

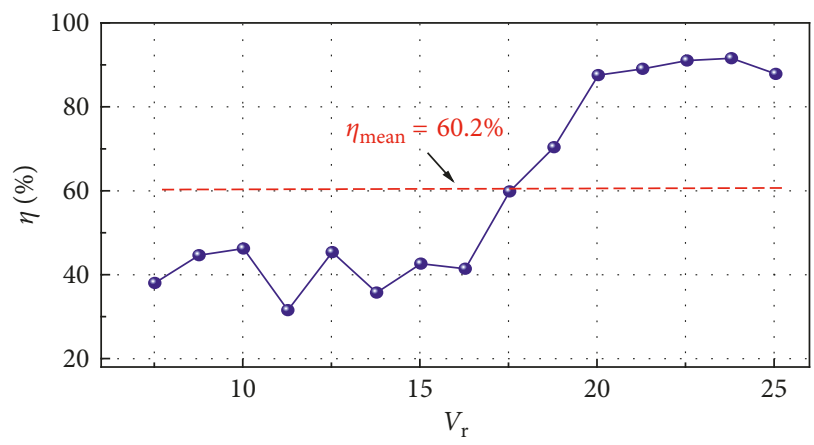

FIGURE 7: VIV suppression efficiencies of the main vertical flexible cylinder with control rods versus the reduced velocity.

can effectively mitigate the CF VIV response of the vertical cylinder.

Figure 8 shows the dominant frequencies and dominant modes of both the smooth cylinder and suppressed cylinder with respect to the reduced velocity. The grey dotted line corresponds to the Strouhal number of a fixed rigid cylinder. It can be observed from Figure 8(a) that for the smooth cylinder, the CF dominant frequency grows linearly with the reduced velocity. A similar trend is also observed in the variation of dominant frequency on the suppressed cylinder, while the data of the suppressed cylinder are slightly lower than that of the smooth cylinder, indicating that the existence of control rods can significantly suppress the dominant frequency of the vertical cylinder. In addition, the dominant mode of the smooth cylinder gradually increases from the first-order to third-order with the increasing reduced velocity (Figure $8(\mathrm{~b})$ ). For the main cylinder with the control rods, the dominant mode shares a similar trend and approximate values with those of the smooth cylinder at all range of the reduced velocity. This phenomenon indicates that the suppressing effectiveness of control rods on the dominant vibration mode of the vertical cylinder is insignificant, which is coincident with the previous experiment result of $\mathrm{Wu}$ et al. $[18,19]$.

3.2. VIV Suppression of the Inclined Flexible Cylinder $\left(\alpha=45^{\circ}\right)$. Figure 9 presents an example of the time-varying strain response and corresponding spectra for the inclined cylinder 


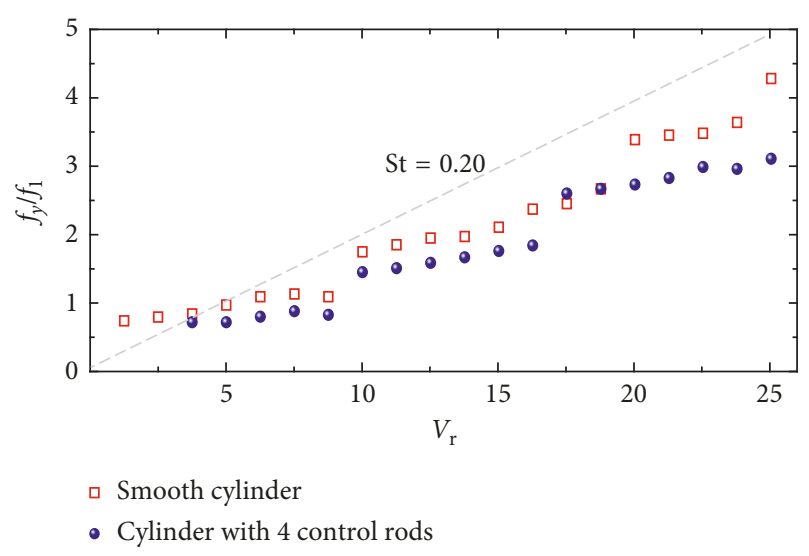

(a)

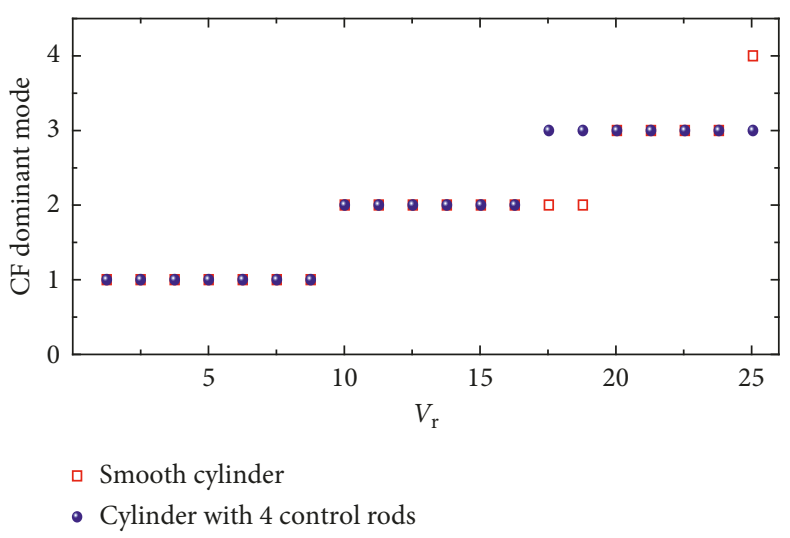

(b)

Figure 8: Dominant frequencies (a) and dominant modes (b) of the main vertical flexible cylinder with control rods versus the reduced velocity.

with $\alpha=45^{\circ}$. To conduct the comparison with the vertical case, $U=0.85 \mathrm{~m} / \mathrm{s}$ is selected. The vertical projected component of the flow velocity $(U \cos \alpha)$ is approximately $0.60 \mathrm{~m} / \mathrm{s}$, which is equal to the flow velocity in the experiment of the vertical cylinder. It can be found that the maximum CF strain of the inclined smooth cylinder can reach approximately $206 \mu \varepsilon$. In addition, the strain amplitude at the middle measurement point G4 is obviously lower than other points and the maximum value appears at locations G2 and G6. The dominant frequency of the inclined smooth cylinder is $5.76 \mathrm{~Hz}$, which corresponds to the 2nd mode. At the same time, a higher harmonic component of approximately $18.1 \mathrm{~Hz}$ is motivated as well. In contrast, the strain and response spectra of the inclined cylinder fitted with control rods are obviously different. The peak value of the strain at measurement points is remarkably suppressed to approximately $45 \mu \varepsilon$, which is an even more satisfying suppressing effectiveness compared to that of the vertical cylinder fitted with control rods. Meanwhile, the response spectra become relatively broadbanded with one lower peak value $(4.53 \mathrm{~Hz})$ by using the control rods for VIV reduction of the inclined cylinder. This phenomenon indicates that the regular vortex shedding of the $45^{\circ}$ inclined cylinder is obviously disrupted. These findings are very meaningful, since previous studies have shown that the VIV suppression effectiveness of helical strakes will deteriorate as the inclination angle increases, and the helical strakes can even reversely enhance the vibration on the inclined flexible cylinder at $45^{\circ}$ inclination [16]. However, the results we present above prove the VIV suppressing reliability of control rods for the inclined flexible cylinder with a large inclination angle.

Figure 10 shows the displacement response characteristic of the $45^{\circ}$ inclined cylinders both without and with control rods. For the inclined smooth cylinder, it can be observed that the amplitude of the time-varying displacement responses is approximately $2.7 \mathrm{D}$ and is observed at measurement points G2 and G6, while the displacement amplitude at the middle measurement point G4 is the lowest. The distribution curve of the maximum RMS displacement of the inclined smooth cylinder illustrates that the CF VIV response is dominated by the 2 nd mode. In contrast, the time-varying displacement response of the $45^{\circ}$ inclined suppressed cylinder shows obvious declination, and the peak value of CF RMS displacement falls to approximately 1.15D. From the spatial distribution plot, it can also be seen that the distribution curve of the max RMS displacement transfers to a smooth parabola, indicating that the vibration is dominated by the 1 st mode. These results above prove the adaptability of multiple control rods on suppressing the CF vibration displacement of the $45^{\circ}$ inclined cylinder.

Figure 11 shows the maximum RMS CF displacements of the smooth and suppressed cylinders versus the reduced velocity for the $45^{\circ}$ inclined case. For the inclined smooth cylinder, the maxima of the RMS CF displacements is nearly $1.48 D$ at $V_{\mathrm{r}}=10.62$. The counterpart of the cylinder with control rods maintains around a lower level. The peak value of CF displacement of the suppressed cylinder reaches approximately $0.57 \mathrm{D}$. Additionally, in terms of the suppression efficiency of the multiple control rods, Figure 12 shows that the efficiency of control rods for the $45^{\circ}$ inclined cylinder appears to be less fluctuating compared with the vertical cylinder, ranging from $37.6 \%$ to $82.3 \%$. No negative efficiency is observed, and the average suppression efficiency can reach up to $67.3 \%$, which is slightly better compared with that of the vertical cylinder, as shown in Figure 7. This result further proves that the inclination of the main body has no negative influence on the VIV suppression effectiveness of control rods, and the control rods can effectively mitigate the CF VIV response on the flexible cylinder with a large inclination angle. The performance of control rods for VIV reduction of an inclined cylinder is obviously different from that of helical strakes in [16]. It was pointed out that the combined actions from the axial flow component and the axial whirling motions result in worse VIV reduction effectiveness of the helical strakes as the increasing inclination angle [16]. However, when the control rods are fitted with the main cylinder, the axial flow cannot whirl along the axis of the cylinder. Hence, the difference of the helical 


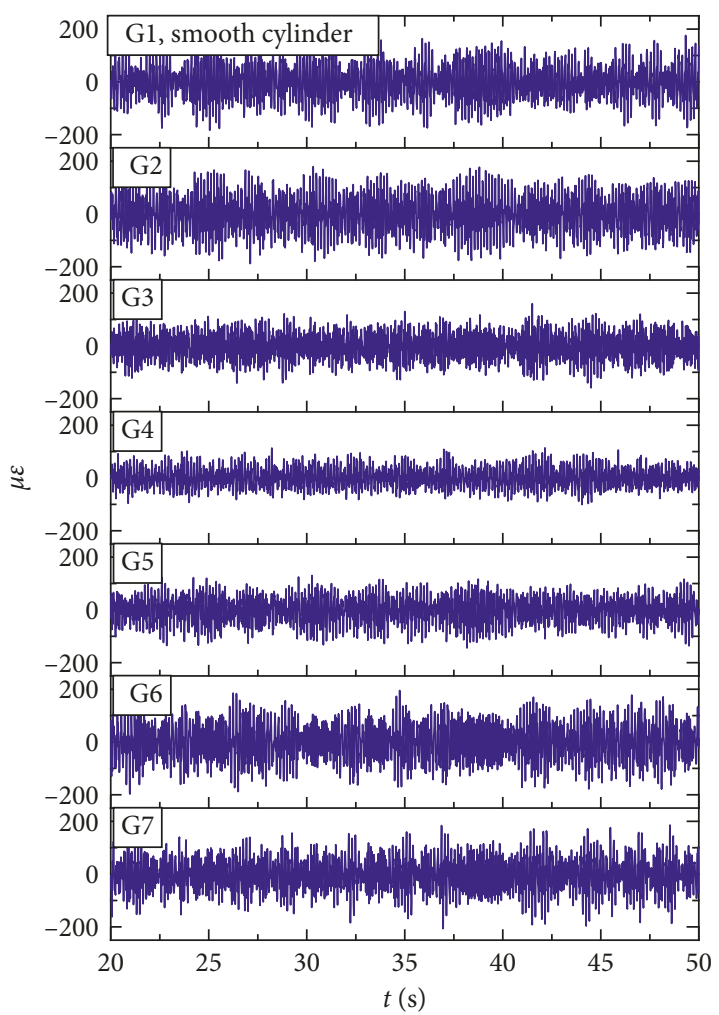

(a)

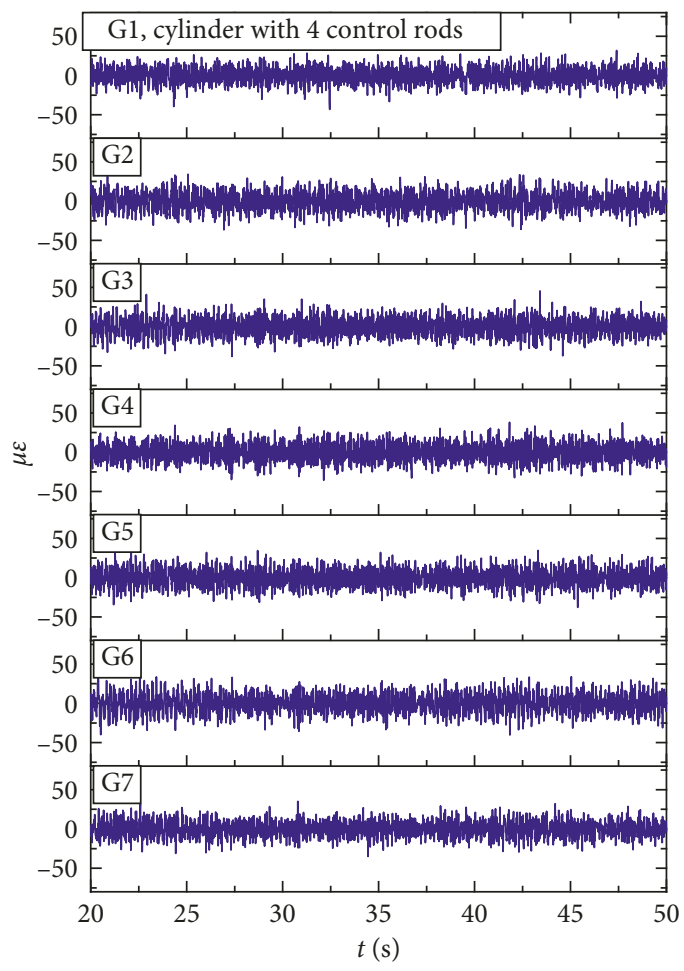

(c)

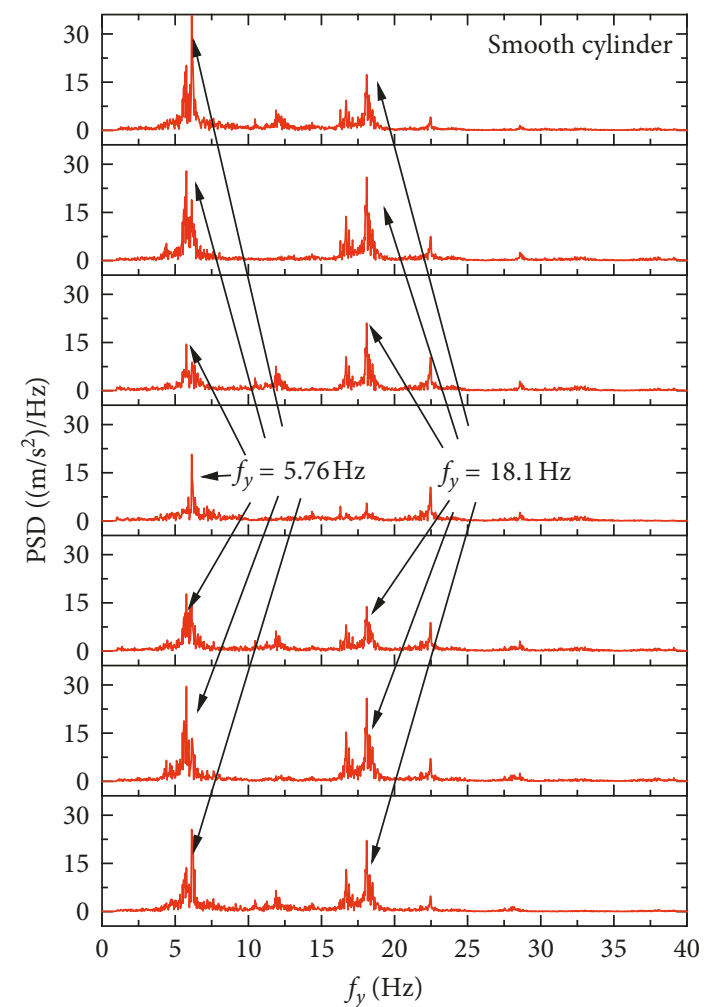

(b)

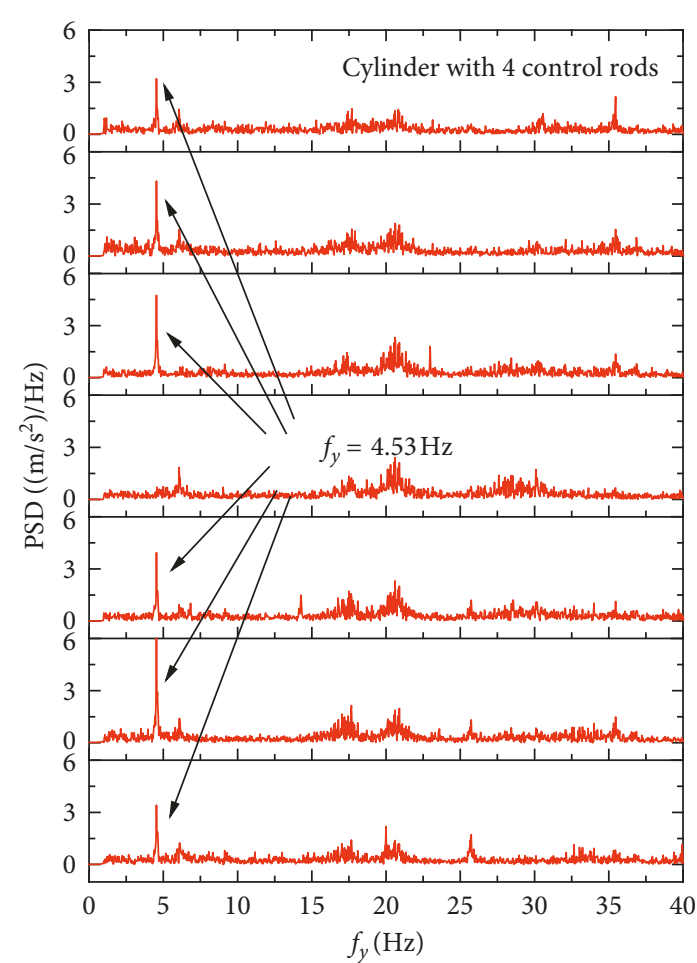

(d)

FIGURE 9: Strain response characteristic of the inclined flexible cylinder $\left(\alpha=45^{\circ}\right)$ without and with control rods undergoing vortex shedding at $U=0.85 \mathrm{~m} / \mathrm{s}$ (the first column presents time history of strain signals $((\mathrm{a}),(\mathrm{c})$ ) and the second column presents response spectra ((b), (d))). 


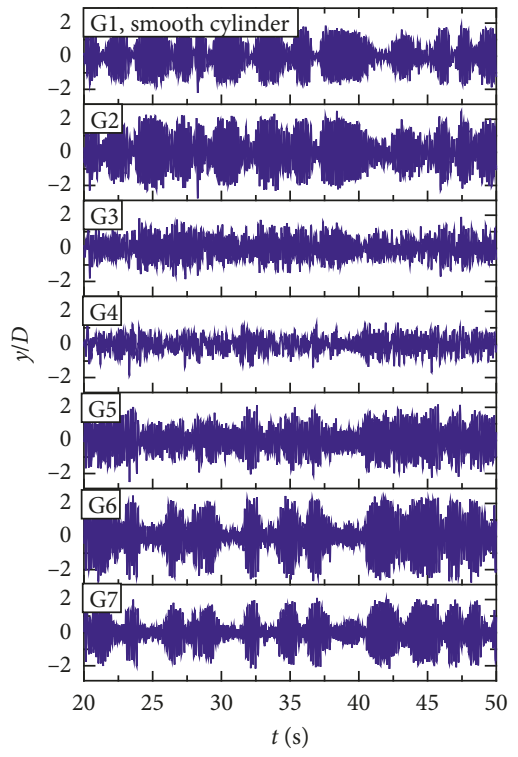

(a)

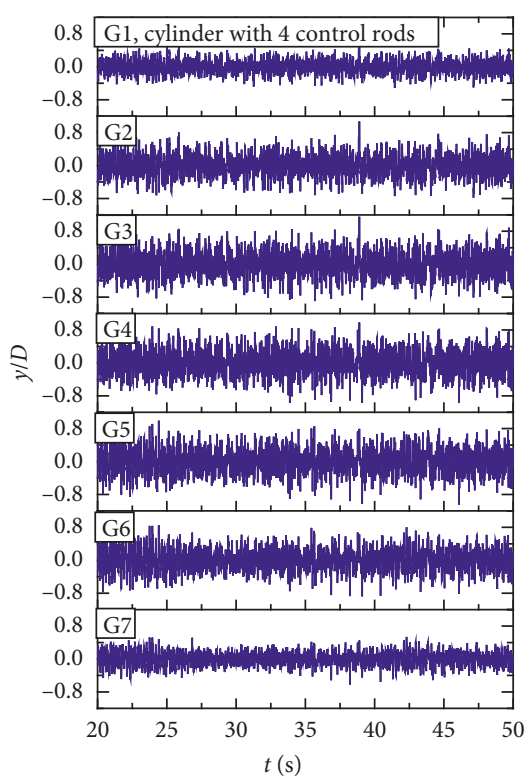

(c)

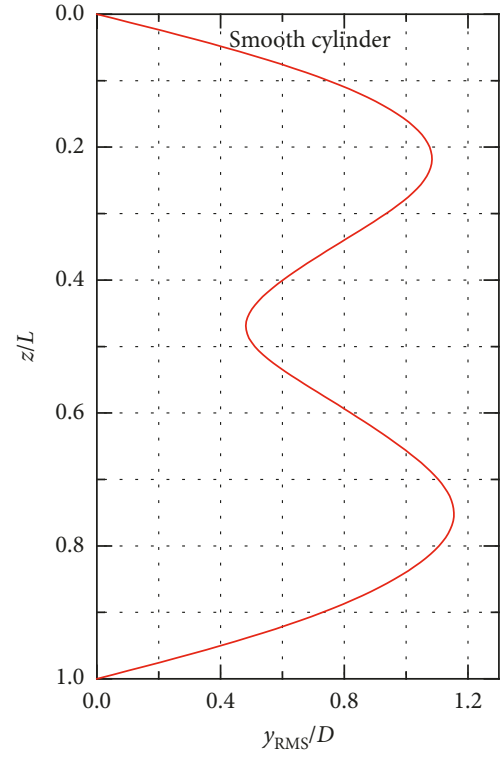

(b)

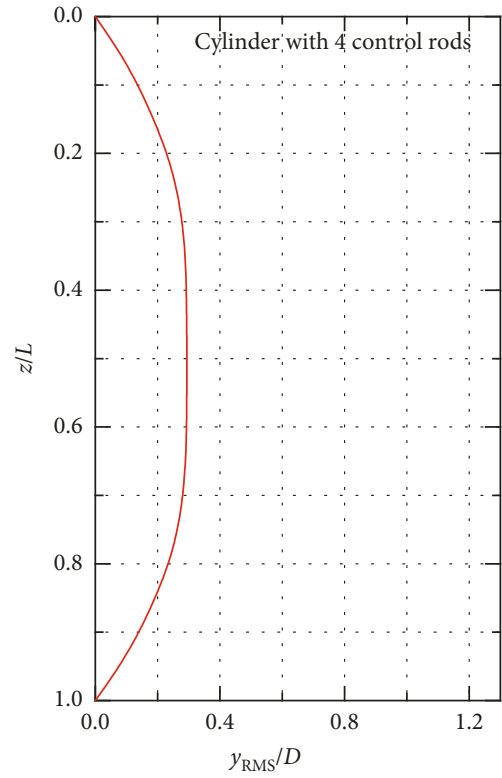

(d)

FIGURE 10: Displacement response characteristic of the inclined flexible cylinder $\left(\alpha=45^{\circ}\right)$ without and with control rods undergoing vortex shedding at $U=0.85 \mathrm{~m} / \mathrm{s}$ (the first column presents the time history of the nondimensional displacement ((a), (c)), the second column presents the spanwise evolutions of the RMS displacements ((b), (d))).

strakes and control rods for VIV suppression of an inclined cylinder is attributed to the influence of the axial flow.

Figure 13 shows that variations of the dominant frequency and dominant mode of the inclined cylinder $(\alpha=$ $45^{\circ}$ ) versus the reduced velocity. The dominant frequency of the inclined smooth cylinder keeps going up with the reduced velocity. The counterpart of the inclined suppressed cylinder shows a similar variation trend but the corresponding data of the suppressed cylinder are slightly lower. This is in accordance with the situation in the vertical test condition, and it indicates that multiple control rods have a positive effect in mitigating the $\mathrm{CF}$ vibration dominant frequency of the inclined cylinder. Furthermore, the CF dominant modes of both the smooth and suppressed cylinders share comparable data and range from the 1st to $3 \mathrm{rd}$ mode. The distinction between the two counterparts at the same reduced velocity is insignificant, which is in line with the experimental results on vertical cylinders. This indicates that the control rods have little influence on the CF dominant mode of the $45^{\circ}$ inclined main cylinder. 


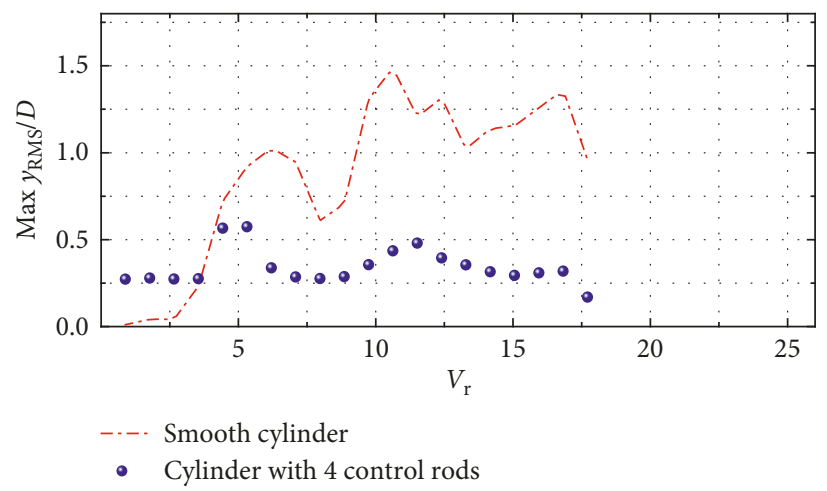

FIGURE 11: Maximum RMS dimensionless displacement of the main inclined flexible cylinder with control rods versus the reduced velocity.

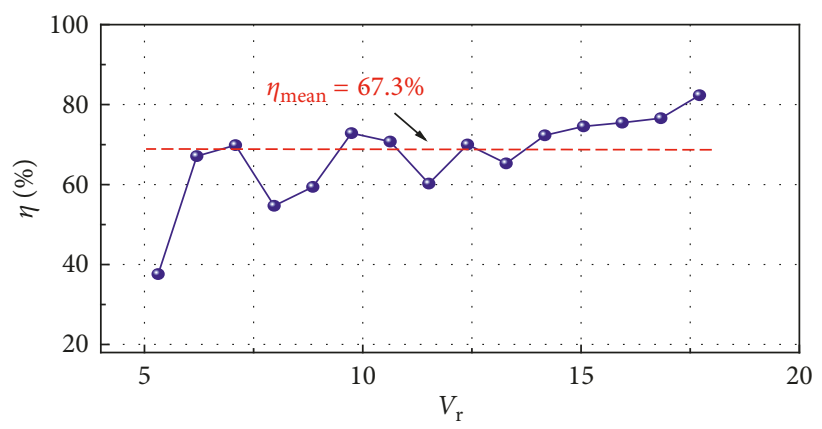

FIGURE 12: VIV suppression efficiencies of the main inclined flexible cylinder with control rods versus the reduced velocity.

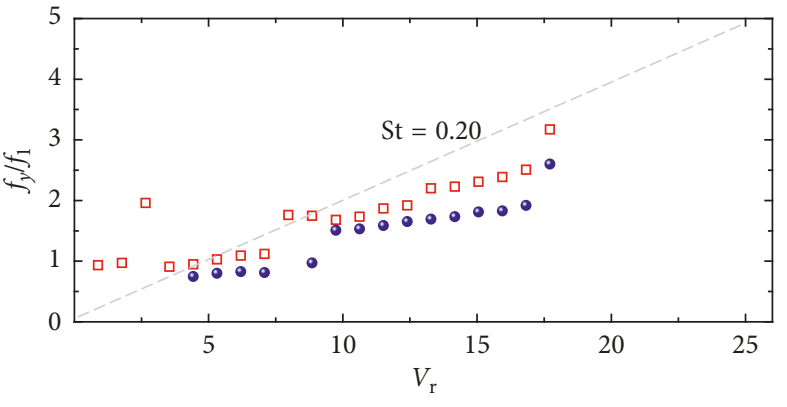

- Smooth cylinder

- Cylinder with 4 control rods

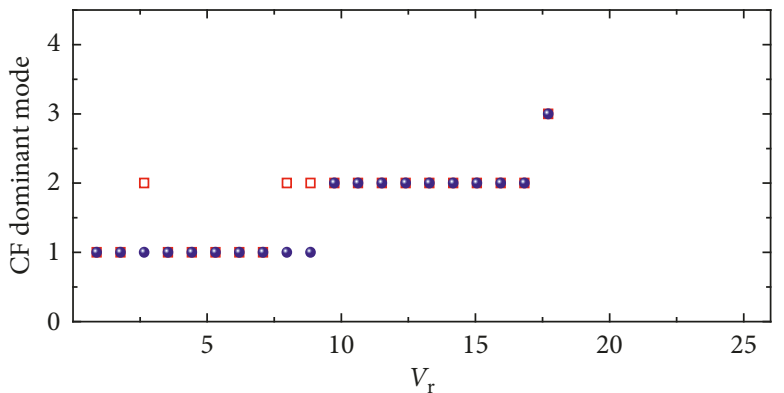

口 Smooth cylinder

- Cylinder with 4 control rods

(b)

Figure 13: Dominant frequencies (a) and dominant modes (b) of the main inclined flexible cylinder with control rods versus the reduced velocity.

\section{Conclusions}

The VIV suppression effectiveness of control rods on the inclined flexible cylinder is experimentally studied in this paper. The cylinder models without and with control rods were towed in the towing tank at two inclination angle cases ( $a=0^{\circ}$ and $45^{\circ}$ ). It is found that the control rods can effectively alleviate the CF VIV response of the vertical flexible cylinder. By attaching the control rods, the peak value of CF strain for the main vertical cylinder decreases from $220 \mu \varepsilon$ to approximately $69 \mu \varepsilon$, and the multiple frequency phenomena disappears. These trends indicate that the control rods can effectively suppress the VIV, which is consistent with previous studies of $\mathrm{Wu}$ et al. [18, 19]. The VIV suppression effectiveness of the control rods is not sensitive to the inclination angle of the main cylinder, and the control rods still keep remarkable performance in suppressing the CF VIV responses on the $45^{\circ}$ inclined flexible cylinder. The suppression effectiveness is similar with that of the vertical cylinder. In addition, the average suppression efficiency of the control rods can reach $67.3 \%$ on the inclined cylinder, which is higher than the counterpart on the vertical cylinder. 
These findings are important to the practical engineering since previous study [16] has found that the VIV suppression effect of another widely used device, helical strake, will deteriorate and even enhance the vibration as the inclination angle increases to $45^{\circ}$.

In addition, more comprehensive and systematic studies should be done in subsequent work. The arrangements of control rods and the inclination angles can be refined, to further investigate the general law of the suppression effectiveness and optimized configuration of the control rods on the inclined flexible cylinder subjected to VIV.

\section{Data Availability}

The data used to support the findings of this study are included in the article.

\section{Conflicts of Interest}

The authors declare that they have no conflicts of interest.

\section{Acknowledgments}

This work was financially supported by the National Natural Science Foundation of China (nos. 51679167, 51778411, and 51678548).

\section{References}

[1] M. M. Zdravkovich, Flow Around Circular Cylinders, Vol. 1: Fundamentals, Vol. 1, Oxford University Press, Oxford, UK, 1997.

[2] C. H. K. Williamson and R. Govardhan, "Vortex-induced vibrations," Annual Review of Fluid Mechanics, vol. 36, no. 1, pp. 413-455, 2004.

[3] R. D. Blevins, Flow-Induced Vibration, Krieger Publishing Inc., Malabar, FL, USA, 2nd edition, 2001.

[4] X. Wu, F. Ge, and Y. Hong, "A review of recent studies on vortex-induced vibrations of long slender cylinders," Journal of Fluids and Structures, vol. 28, pp. 292-308, 2012.

[5] M. M. Zdravkovich, "Review and classification of various aerodynamic and hydrodynamic means for suppressing vortex shedding," Journal of Wind Engineering and Industrial Aerodynamics, vol. 7, no. 2, pp. 145-189, 1981.

[6] P. W. Bearman and J. C. Owen, "Reduction of bluff-body drag and suppression of vortex shedding by the introduction of wavy separation lines," Journal of Fluids and Structures, vol. 12, no. 1, pp. 123-130, 1998.

[7] H. Choi, W. P. Jeon, and J. Kim, "Control of flow over a bluff body," Annual Review of Fluid Mechanics, vol. 40, no. 1, pp. 113-139, 2008.

[8] R. Galvao, E. Lee, D. Farrell et al., "Flow control in flowstructure interaction," Journal of Fluids and Structures, vol. 24, no. 8, pp. 1216-1226, 2008.

[9] G. R. S. Ássi, P. W. Bearman, and N. Kitney, "Low drag solutions for suppressing vortex-induced vibration of circular cylinders," Journal of Fluids and Structures, vol. 25, no. 4, pp. 666-675, 2009.

[10] Y. Gao, S. X. Fu, J. Cao, and Y. E. Chen, "Experimental study on response performance of VIV of a flexible riser with helical strakes," China Ocean Engineering, vol. 29, no. 5, pp. 673-690, 2015.

[11] Y. Gao, S. X. Fu, T. Ren, Y. M. Xiong, and L. J. Song, "VIV response of a long flexible riser fitted with strakes in uniform and linearly sheared currents," Applied Ocean Research, vol. 52, pp. 102-114, 2015.

[12] Y. Gao, J. D. Yang, Y. M. Xiong, and M. H. Wang, "Experimental investigation of the effects of the coverage of helical strakes on the vortex-induced vibration response of a flexible riser," Applied Ocean Research, vol. 59, pp. 53-64, 2016.

[13] Norwegian Marine Technology Research Institute, Marintek Review No.1-April 2005: Systematic Investigation of Efficiency of Helical Strakes, Marintek, Trondheim, Norway, 2005.

[14] T. Zhou, S. F. M. Razali, and Z. Hao, "On the study of vortexinduced vibration of a cylinder with helical strakes," Journal of Fluids and Structures, vol. 27, no. 7, pp. 903-917, 2011.

[15] L. Lee, D. W. Allen, and D. L. Henning, "Damping characteristics of fairings for suppressing vortex-induced vibrations," in Proceedings of 23rd International Conference on Offshore Mechanics and Arctic Engineering, pp. 433-438, Vancouver, BC, Canada, January 2004.

[16] W. H. Xu, Y. S. Luan, and Q. H. Han, "The effect of yaw angle on VIV suppression for an inclined flexible cylinder fitted with helical strakes," Applied Ocean Research, vol. 67, pp. 263-276, 2017.

[17] S. J. Lee, S. I. Lee, and C. W. Park, "Reducing the drag on a circular cylinder by upstream installation of a small control rod," Fluid Dynamics Research, vol. 34, no. 4, pp. 233-250, 2004.

[18] H. Wu, D. Sun, and L. Lu, "Influence of attack angle on VIV suppression by multiple control rods for long flexible riser model," in Proceedings of International Offshore and Polar Engineering Conference, Maui, HI, USA, June 2011.

[19] H. Wu, D. Sun, and L. Lu, "Experimental investigation on the suppression of vortex-induced vibration of long flexible riser by multiple control rods," Journal of Fluids and Structures, vol. 30, no. 2, pp. 115-132, 2012.

[20] M. Silva-ortega and G. R. S. Assi, "Flow-induced vibration of a circular cylinder surrounded by two, four and eight wakecontrol cylinders," Experimental Thermal and Fluid Science, vol. 85, pp. 354-362, 2017.

[21] P. F. Zhang, J. J. Wang, and L. X. Huang, "Numerical simulation of flow around cylinder with an upstream rod in tandem at low Reynolds numbers," Applied Ocean Research, vol. 28, no. 3, pp. 183-192, 2006.

[22] H. Zhu and J. Yao, "Numerical evaluation of passive control of VIV by small control rods," Applied Ocean Research, vol. 51, pp. 93-116, 2015.

[23] H. Zhu, J. Yao, and Y. Ma, "Simultaneous CFD evaluation of VIV suppression using smaller control cylinders," Journal of Fluids and Structures, vol. 57, pp. 66-80, 2015.

[24] W. H. Xu, Y. X. Ma, C. N. Ji, and C. Sun, "Laboratory measurements of vortex-induced vibrations of a yawed flexible cylinder at different yaw angles," Ocean Engineering, vol. 154, pp. 27-42, 2018.

[25] J. R. Chaplin, P. W. Bearman, F. J. Huera-Huarte, and R. J. Pattenden, "Laboratory measurements of vortex-induced vibrations of a vertical tension riser in a stepped current," Journal of Fluids and Structures, vol. 21, no. 1, pp. 3-24, 2005.

[26] A. D. Trim, H. Braaten, H. Lie, and M. A. Tognarelli, "Experimental investigation of vortex-induced vibration of long marine risers," Journal of Fluids and Structures, vol. 21, no. 3, pp. 335-361, 2005. 
[27] H. Lie and K. E. Kaasen, "Modal analysis of measurements from a large-scale VIV model test of a riser in linearly sheared flow," Journal of Fluids and Structures, vol. 22, no. 4, pp. 557-575, 2006

[28] J. N. Song, L. Lu, B. Teng, H. I. Park, G. Q. Tang, and H. Wu, "Laboratory tests of vortex-induced vibrations of a long flexible riser pipe subjected to uniform flow," Ocean Engineering, vol. 38, no. 11-12, pp. 1308-1322, 2011. 


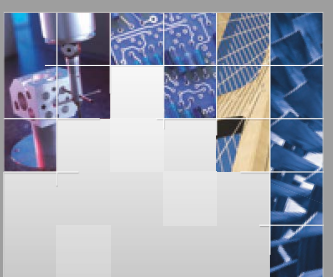

\section{Enfincering}
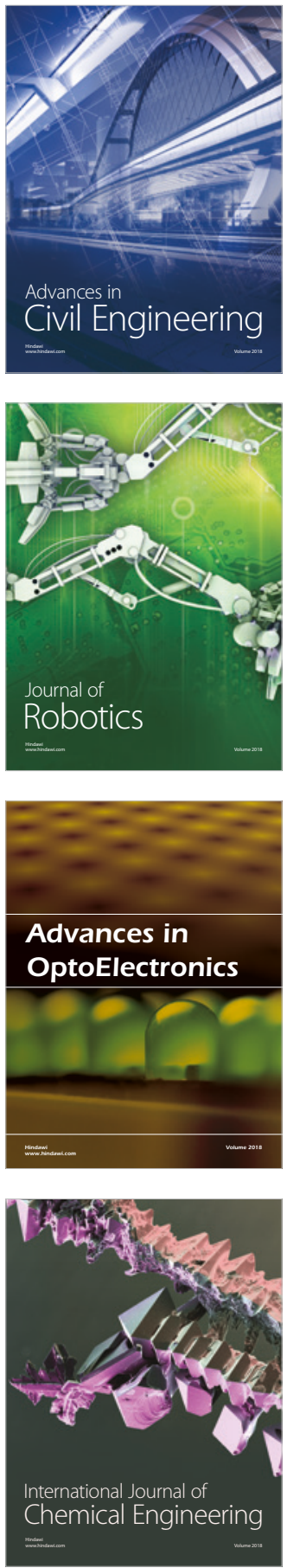

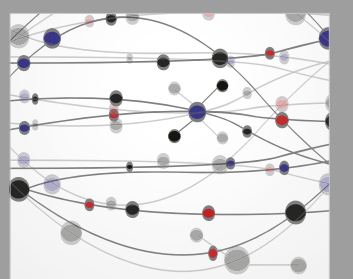

\section{Rotating \\ Machinery}

The Scientific World Journal

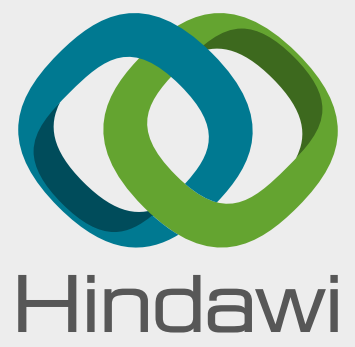

Submit your manuscripts at

www.hindawi.com
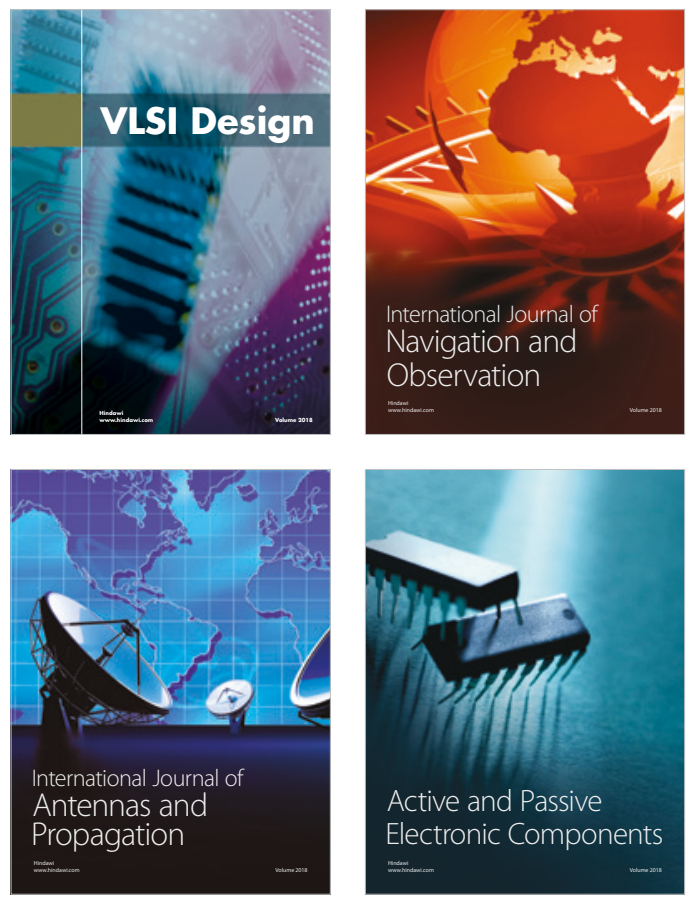
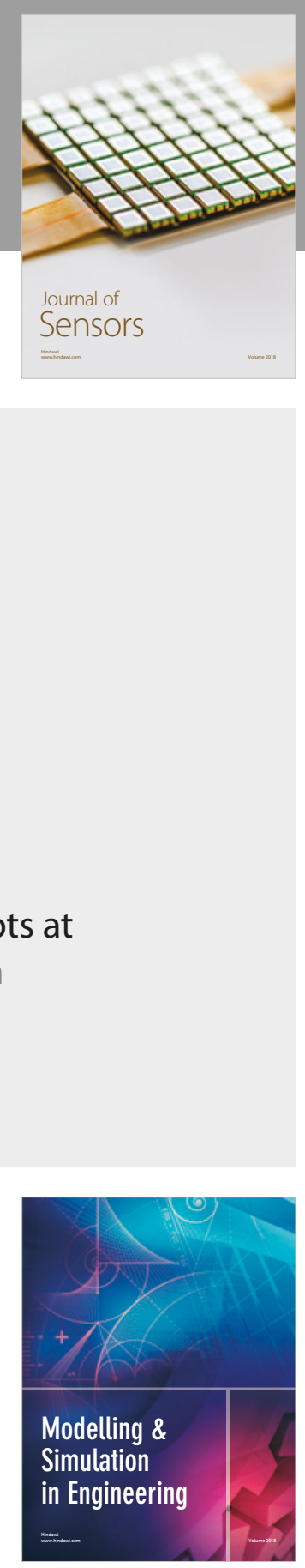

\section{Advances \\ Multimedia}
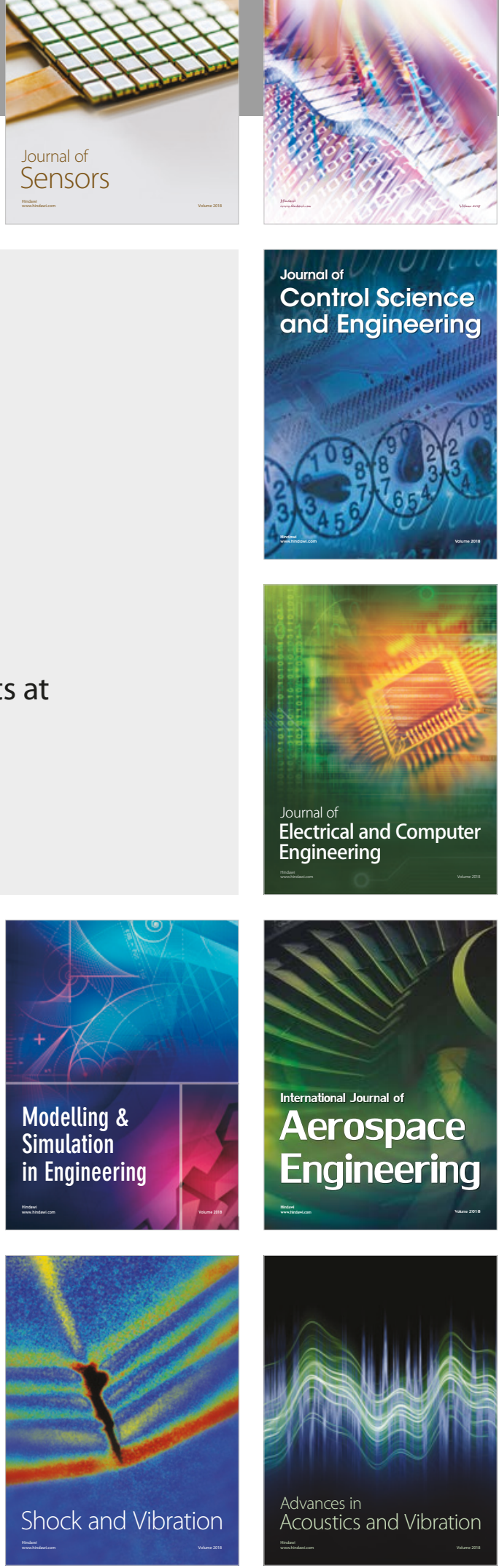